\title{
Correction: Oveisgharan, S., et al. Evaluating the Preconditions of Two Remote Sensing SWE Retrieval Algorithms over the US, Remote Sensing 2020, 12, 2021
}

\author{
Shadi Oveisgharan ${ }^{1, *}$, Daniel Esteban-Fernandez ${ }^{1}$, Duane E. Waliser ${ }^{1}$, Randall R Friedl ${ }^{1}$, \\ Son V. Nghiem ${ }^{1}$ and Xubin Zeng ${ }^{2}$ \\ 1 Jet Propulsion Lab, Pasadena, CA 91109, USA; daniel.esteban-fernandez@jpl.nasa.gov (D.E.-F.); \\ duane.e.waliser@jpl.nasa.gov (D.E.W.); randall.r.friedl@jpl.nasa.gov (R.R.F.); \\ son.v.nghiem@jpl.nasa.gov (S.V.N.) \\ 2 Department of Atmospheric Sciences, University of Arizona, Tucson, AZ 85719, USA; \\ xubin@email.arizona.edu \\ * Correspondence: Shadi.Oveisgharan@jpl.nasa.gov
}

Received: 28 July 2020; Accepted: 11 August 2020; Published: 12 August 2020

check for updates

The authors would like to add Radar to the title for more clarification.

Evaluating the Preconditions of Two Radar Remote Sensing Retrieval Algorithms Over the US.

The authors also wish to make the following editorial corrections to the body of the paper.

1. On page 3, line 17, the sentence "We requested the dataset directly from the University of Arizona so we can had other layers of the dataset,... " should be "We requested the dataset directly from the University of Arizona so we can have other layers of the dataset, ... ".

2. On page 7, the second line of the last paragraph, the sentence "This means that there are relatively few grid points with lots of SWEs that do not satisfy the precondition of dual-pol. dual. freq. retrieval algorithm." should be "This means that there are relatively few grid points with many values of SWEs that do not satisfy the precondition of dual-pol. dual. freq. retrieval algorithm.".

3. On page 14, line 8, the sentence "More studies are needed to better model temporal coherence at different frequencies, different land types, and different environmental condition" should be "More studies are needed to better model temporal coherence at different frequencies, different land types, and different environmental conditions".

4. On page 14, line 29, the sentence "Our analysis here are based on differential interferometry retrieval algorithm not single-pass interferometry." should be "Our analysis here is based on differential interferometry retrieval algorithm not single-pass interferometry.".

5. On page 14 (bottom of page), by removing $i$ at the end of the sentence "Differential interferometric phase retrieval algorithm is not applicable to the snow with penetration depth smaller than snow depth $i$ " should be "Differential interferometric phase retrieval algorithm is not applicable to the snow with penetration depth smaller than snow depth".

6. On page 15, line 1, the sentence "is considered for retrieval algorithm to avoid phase unwrapping problem" should be "is considered for the retrieval algorithm to avoid phase unwrapping problem".

7. On page 15 , line 10 , by adding degrees, the sentence "We assume the incidence angle is 30 " should be "We assume the incidence angle is 30 degrees".

8. On page 16 , line 7 , the sentence "the percentage of points suitable for differential interferometry retrieval method is larger in 2015 compared to 1997" should be "the percentage of points suitable for the differential interferometry retrieval method is larger in 2015 compared to 1997".

9. On page 18 , line 2 in conclusions, the sentence "In this study we identify the regions and the amount of snow in the US that satisfy the pre-condition of the two major SWE retrieval algorithms." 
should be "In this study we identify the regions and the amount of snow in the US that satisfy the pre-condition of the two candidate radar-based SWE retrieval algorithms.".

The authors would like to apologize for any inconvenience caused to the readers by these changes.

\section{Reference}

1. Oveisgharan, S.; Esteban-Fernandez, D.; Waliser, D.; Friedl, R.; Nghiem, S.; Zeng, X. Evaluating the Preconditions of Two Remote Sensing SWE Retrieval Algorithms over the US. Remote Sens. 2020, 12, 2021. [CrossRef]

(C) 2020 by the authors. Licensee MDPI, Basel, Switzerland. This article is an open access article distributed under the terms and conditions of the Creative Commons Attribution (CC BY) license (http://creativecommons.org/licenses/by/4.0/). 